Robb et al.: Biomarkers and ADL in AD

\title{
Biomarkers and functional decline in prodromal Alzheimer's disease
}

Catherine Robb MSc ${ }^{a \dagger}$, Chinedu Udeh-Momoh $\mathrm{PhD}^{\mathrm{a}, \mathrm{b} \dagger}$, Stefan Wagenpfeil $\mathrm{PhD}^{\mathrm{c}}$, Jakob Schöpe $\mathrm{MSc}^{\mathrm{c}}$, Panagiotis Alexopoulos MD $\mathrm{d}^{\mathrm{d}, \mathrm{e} \dagger}$ and Robert Perneczky MD $\mathrm{D}^{\dagger \dagger \# \mathrm{a}, \mathrm{d}, \mathrm{f,g}}$ for the Alzheimer's Disease Neuroimaging Initiative*

$\dagger$ These two authors contributed equally to the manuscript.

${ }^{\dagger \dagger}$ These two authors contributed equally to the manuscript.

${ }^{a}$ Neuroepidemiology and Ageing Research Unit, School of Public Health, Faculty of Medicine, The Imperial College of Science, Technology and Medicine, London, UK

${ }^{b}$ MRC Centre for Synaptic Plasticity, School of Clinical Sciences, University of Bristol, Bristol, UK

${ }^{\mathrm{c}}$ Institute for Medical Biometry, Epidemiology and Medical Informatics, Universität des Saarlandes, Campus Homburg, Germany

${ }^{\mathrm{d}}$ Department of Psychiatry and Psychotherapy, Ludwig-Maximilians-Universität München, Munich, Germany

${ }^{\mathrm{e}}$ Department of Psychiatry, University of Patras, Rion Patras, Greece

${ }^{\mathrm{f}}$ West London Mental Health NHS Trust, London, UK

${ }^{\mathrm{g}}$ German Center for Neurodegenerative Diseases (DZNE) Munich, Munich, Germany 
Robb et al.: Biomarkers and ADL in AD

* Data used in preparation of this article were obtained from the Alzheimer's Disease

Neuroimaging Initiative (ADNI) database (adni.loni.usc.edu). As such, the investigators within the ADNI contributed to the design and implementation of ADNI and/or provided data but did not participate in analysis or writing of this report. A complete listing of ADNI investigators can be found at: http://adni.loni.usc.edu/wp-

content/uploads/how_to_apply/ADNI_Acknowledgement_List.pdf

${ }^{\#}$ Correspondence address:

Prof Robert Perneczky

Department of Psychiatry and Psychotherapy

Ludwig-Maximilians-Universität München

Nußbaumstr. 7, 81679 München

Tel: +49894400 55772, Fax: +4989440053413

Email: robert.perneczky@med.uni-muenchen.de 
Robb et al.: Biomarkers and ADL in AD

\section{ABSTRACT}

BACKGROUND: Little is known of possible associations between Alzheimer's disease (AD) biomarkers and instrumental activities of daily living (IADL) change over time.

OBJECTIVE: The present study seeks to identify relationships between baseline imaging and fluid biomarker profiles, and decline in IADL utilising data collated from the AD Neuroimaging Initiative (ADNI) cohort.

METHODS: Generalised estimating equations analysis, adjusted for cognitive deterioration, was applied to a cohort of 509 individuals from all stages of ADNI, including 156 healthy controls, 189 early mild cognitive impairment (MCI) patients and 164 MCI patients.

RESULTS: A significant correlation was found between baseline biomarkers - specifically CSF $\mathrm{A} \beta$ and FDG PET, and IADL change over a 3-year period in individuals with MCI. Importantly, comparable correlations between presence of pathological biomarker levels and temporal decline in both functional and cognitive performance were also noted.

DISCUSSION: We show that distinct baseline biomarkers may predict latent changes in IADL. Our results necessitate a revision of the commonly held view upholding cognitive changes as the predominant endpoint measure associated with presence of abnormal baseline biomarkers.

Keywords: Alzheimer's disease, biomarker, prediction, early diagnosis, activities of daily living, cerebrospinal fluid, positron emission tomography, magnetic resonance imaging 


\section{Introduction}

The clinical stages of Alzheimer's disease (AD) are characterised by a slowly progressive memory-dominant cognitive deterioration, which is accompanied by a progressive impairment of normal instrumental activities of daily living (IADL) [1,2] including complex activities such as managing finances, driving or using public transportation, undertaking household chores or doing the shopping [3]. Presence of early cognitive, and IADL changes are evident up to 10 years before an individual meets the diagnostic criteria for $\mathrm{AD}$ dementia [4], where evidence of significant cognitive and functional deterioration is required to establish AD diagnosis [5].

Until recently the in vivo diagnosis of AD was mainly based on clinical judgement, however newly proposed guidelines emphasise the use of biomarkers such as cerebrospinal fluid (CSF) and neuroimaging measures to identify the presence of AD pathology at different disease stages, as well as predict the progression from the earliest symptomatic stage, i.e. early mild cognitive impairment (eMCI) [6], to AD dementia [7, 8]. Although future cognitive decline can be predicted with relatively high accuracy using certain neuropsychological tests, established CSF, imaging and genetic biomarkers: total-Tau (t-tau), pospho-Tau 181 (p-tau) and amyloid- $\beta_{1-42}(\mathrm{~A} \beta 1-$ 42), magnetic resonance imaging (MRI) mediotemporal lobe atrophy, 18F-fluorodeoxyglucose (FDG) positron-emission-tomography (PET) glucose metabolism, C11-Pittsburgh-Compound-B (PIB) PET fibrillar amyloid load and apolipoprotein- $\varepsilon 4$ allele (APOE- $\varepsilon 4)$ [9-11], little is known about the association between presence of AD risk-biomarkers and IADL change over time.

It is well established that functional decline is commonly used as a co-primary endpoint in many clinical studies, along with cognitive decline [7, 8]. In addition, biomarkers are commonly used as surrogate endpoints in clinical drug trials, and have provided strong evidence of predicting cognitive decline over time. Recent Food and Drug Administration (FDA) guidelines [12] for AD clinical trials designed to capture participants at earlier disease stages, have asked that a combined cognitive and functional co-primary outcome measure approach be applied to demonstrate 
efficacy, and ensure clinical meaningfulness of a cognitive benefit observed through drug treatment. Even though functional decline is considered an equally important and highly clinically relevant co-outcome measure, there is still very little evidence to show that baseline biomarker measures could serve as predictors of function decline (independent of cognitive decline). In order to reliably utilise AD biomarkers as surrogate endpoints in clinical trials involving functional and cognitive co-endpoint measures, it is critical to ascertain whether baseline biomarkers can indeed reliably predict functional decline independently of cognitive decline.

Previously published data suggest that early biomarker measures may indicate future IADL regression usually in late prodromal and early stage disease populations [13-16]. However to date, no study has sought to put together a comprehensive picture of how multiple baseline AD biomarker measures may impact on downstream IADL decline, specifically within a prodromal population. Furthermore, it is not clear if baseline biomarkers predict IADL change independently of baseline cognitive status, which itself is a strong factor influencing functional performance. This study utilises data obtained from the AD Neuroimaging Initiative (ADNI) cohort. By analysing data collected from patients in the earliest clinical stages of AD as well as healthy elderly controls; we examined the relationship between baseline imaging and fluid biomarker measures and IADL change over time through comparisons of functional deterioration in individuals with normal versus abnormal baseline biomarker measures.

\section{Materials and methods}

Data used in the preparation of this article were obtained from the ADNI database (adni.loni.usc.edu) on $25^{\text {th }}$ March 2014. The ADNI was launched in 2003 as a public-private partnership, led by Principal Investigator Michael W. Weiner, MD. The primary goal of ADNI has been to test whether serial MRI, PET, plus additional biological markers, clinical and neuropsychological assessment can be combined to measure the progression of MCI and early 
clinical AD. ADNI was reviewed and approved by all host study site review boards, and participants completed informed consent after receiving a comprehensive description of ADNI. Participants were aged between 55-90 (inclusive), considered cognitively normal (CN), eMCI, MCI or AD dementia diagnosed individuals, and underwent serial evaluations of functional, biomedical, neuropsychological and clinical status at various intervals.

\subsection{Study population}

The current study utilised data collected at baseline and 24- to 36-months in 12-month increments. In cases where there were an $\mathrm{N}$ too small to consider at 36-months, follow-ups were only included to 24 -months (review Supplementary Figure $1 \& 2$ ). Included were baseline data from CN subjects (defined as MMSE score between 25 and 30, inclusive; CDR score of 0; no evidence of depression; and no memory complaints; $n=156)$, subjects with eMCI ( $n=189)$, and patients with MCI ( $n=164)$ from all stages of ADNI (ADNI 1 n=199, ADNI GO n=6, ADNI 2 n=377). Both eMCI and MCI were defined as MMSE score between 24 and 30, inclusive; CDR score of 0.5; report of memory complaints; no significant functional impairment. Additionally, to distinguish between the diagnostic groups: eMCI and MCI; objective memory deficits on the Wechsler Memory-Scale-Logical Memory II test was used with scores between 0.5SD-1.5SD depicting eMCI and lower than 1.5SD below the norm indicative of MCI. This ensured no overlap between the diagnostic groups. Included subjects had available structural MRI scans, FDG PET, CSF proteins (A $\beta 1-42$, t-tau and p-tau) and $A P O E-\varepsilon 4$ allele carrier status (dichotomised into carriers vs non-carriers). Of the samples obtained, 113 participants were missing baseline MRI scans, hence, the 3-month follow up visit data was alternatively included. Additionally included were available free recall trial measures from the Rey Auditory Verbal Learning Test (RAVLT) [17] and a measure of IADL, the Functional Activities Questionnaire (FAQ) [18], both being obtained from each of the above mentioned visit times, up to 36-months, where available (Table 1). The RAVLT 
measures verbal memory performance, where the sum of the five RAVLT free recall trials score was used; scores ranging from 0-75; lower scores on the RAVLT indicate greater memory impairment. This tool was chosen for its known sensitivity in measuring early signs of episodic memory decline; a key indicator of MCI due to AD [19-22]. The FAQ is an informant-based measure of IADL. Ratings range from normal (0) to dependent (3) on 10 subscales for a total of 30 points, with higher scores indicating more impaired functional status. An informant based questionnaire of functional performance far exceeds the cogency of a self-reporting version, with FAQ exhibiting highest validity in predicting AD related functional decline in multiple studies [2]. Further rationale for the utilisation of this instrument is reflected in a pioneering study reported by Teng and others, where the authors indicated that FAQ scores $>6$ were consistent with functional impairment [23].

\subsection{Biomarkers}

An in-depth description of biomarker acquisition and performance measures in ADNI can be obtained at www.loni.ucla.edu/ADNI, with image and CSF collection protocols available elsewhere [24-27]. Briefly, TaqMan quantitative polymerase chain reaction assays were used for genotyping APOE nucleotides $334 \mathrm{TC}$ and $472 \mathrm{CT}$ with an ABI 7900 real-time thermocycler (Applied Biosystems, Foster City, CA) using DNA freshly prepared from whole blood samples [28]. Mean FDG count was obtained per subject based on a composite region of interest in an AD typical hypometabolic pattern [24, 26]. FreeSurfer software (http://surfer.nmr.mgh.harvard.edu) was utilised to extract MRI (1.5 T) measured hippocampal volume where an atlas-based approach was implemented and has been validated for use in subjects with a great deal of morphologic variability. Uncorrected hippocampal volume for head size was used as a previous study showed that the association between hippocampal volumes and cognition was not altered by intracranial volume normalization [29]. Peptide CSF measures were generated from aliquot samples collected 
at the same time [30] using commercially available enzyme-linked immunosorbent assays (ELISAs). Validated cut-offs were applied to a differential between normal and pathological findings for CSF p-tau and t-tau [26, 31-32], CSF A $\beta$ [24, 31-33], FDG PET [24-26] and MRI hippocampal volume [24-26, 31-33].

\subsection{Statistical analysis}

Linear generalised estimating equations (GEE) were conducted to examine the influence of baseline biomarker measures on IADL deterioration. The same analysis was utilised to compare and contrast results with cognitive deterioration. In this report, time is represented such that repeated measurements are dependent samples, whereas measurement between patients are considered to be independent. Accordingly, FAQ and RAVLT were modeled as dependent variables, and baseline biomarker measures as independent variables in separate GEE analyses. Baseline biomarker measures where dichotomized into positive (abnormal) and negative (normal) measures, hence our analyses are based on these values and not the original biomarker measurement values. GEE uses all available data from all points in time (including baseline measures) to estimate a population-averaged effect of biomarkers on FAQ/RAVLT over time. GEE does not model the (relative) change of FAQ/RAVLT as a dependent variable but as original values obtained from each individual over time. Concerning the dependent variable, GEE accounts for dependency of observations measured in the same individual over time by using a working correlation structure. Selection of GEE as an appropriate statistical model for this study allowed an estimation of the temporal effect of baseline imaging and CSF AD-biomarker measures presence on IADL across the investigated population (population-averaged effects), as opposed to within subject effects. Additionally, the model took into account missing values across the time course. Additional information on GEE models may be viewed elsewhere [34, 35]. 
Longitudinal analyses were adjusted for age, education and $A P O E \varepsilon 4$-allele status. In addition, FAQ was adjusted for RAVLT at baseline and vice-versa. We did not adjust for gender due to identified studies reporting zero gender bias on variations in IADL performance over time [36]. Statistical two-sided significance level was set at 5\% ( $<<0.05)$. To our knowledge, this study is the first to explore associations between baseline biomarkers and IADL change independently of cognitive decline, therefore our analyses are purely exploratory. Hence, we considered it appropriate not to adjust for multiple comparisons as this is only necessary for confirmatory analyses [37]. All analyses were performed using the gee and ggplot2-packages in R version 3.2.1 [38-40].

\section{Results}

Relevant sample characteristics are presented in Table 1.

A significant association was found between baseline FAQ and RAVLT scores $(\mathrm{p}<0.001)$, where the Spearman's correlation coefficient between baseline FAQ and RAVLT given as $r=-0.40$, however, FAQ and RAVLT scores after baseline were no longer comparable. Significant associations between IADL, cognitive performance and specific biomarkers were found in both eMCI and MCI groups prior to adjustment, but not in the $\mathrm{CN}$ group.

There were no significant associations among the eMCI patient group once results were adjusted for age, $A P O E-\varepsilon 4$ carriage, education and respectively FAQ (GEE models for AVLT) or AVLT (GEE models for FAQ) (Table 2).

In MCI patients, worsening FAQ scores were found to be associated with impaired glucose metabolism [FDG-PET data] $(r=3.59, p<0.001)$ and low levels of CSF A $\beta(r=2.97, p=0.028)$. In addition, RAVLT decline among MCI patients significantly correlated with abnormal glucose metabolism at baseline ( $r=-4.60, p=0.009)$, low CSF A $\beta(r=-6.59, p<0.005)$ and high levels of CSF p-tau $(r=-6.85, p<0.001)$ (Table 2). 
In healthy controls, no significant associations were found between any of the biomarkers and FAQ as well as RAVLT scores.

The associations between baseline biomarkers and FAQ scores for all investigated subjects are presented in Supplementary Figure 1, and for RAVLT in Supplementary Figure 2. The results indicate that FAQ decline is independent of RAVLT decline, as can be seen by the inconsistent coefficients, that is, the signs of the coefficients (RAVLT vs. FAQ) for the same biomarker are neither consensual (same direction; e.g. both positive) nor opposing. Moreover, relative differences between the two scales do not correlate after 12, 24 and 36 months (Spearman's correlation coefficient: $<0.2)$, whereby a high correlation $(<.05)$ between the relative differences (e.g. baseline vs 12-months) of the two scales would suggest a dependence between the FAQ and RAVLT scales.

\section{Discussion}

Identification of alternate clinical study endpoint measures for AD clinical trials remains a crucial endeavour to be pursued, in light of recent FDA guidelines advocating the implementation of a multi-outcome measure approach (utilising not only cognitive, but also functional outcome measures) for a more robust design when executing clinical trials investigating AD drug targets [12]. Although emerging evidence posits that functional decline may serve as an adjunctive primary outcome measure with cognitive deterioration, and results on baseline biomarkers reliably predicting latent cognitive decline are none too few; the data on associations between distinct baseline AD risk biomarkers and temporal changes in IADL remain very limited. In a bid to determine whether baseline biomarkers co-predict changes not only in cognitive, but also functional performance, we sought to explore the association between baseline AD biomarker measures and functional decline independently of cognitive decline over time in healthy, eMCI and MCI elderly individuals. We therefore aimed to observe whether functional decline would 
significantly differ between individuals who present with positive (abnormal) versus negative (normal) biomarker measures at baseline. By utilising a sub-set of participant data from the ADNI cohort, we implemented GEE to critically evaluate notable associations between objective assessments of cognitive (RAVLT) and functional (FAQ) change in relation to distinct AD risk biomarkers collected at subject enrolment.

Our data, adjusted for baseline cognitive performance and APOE $\varepsilon 4$-allele carriage, revealed that functional decline in the MCI subject group was significantly correlated with abnormal glucose metabolism (assessed using FDG-PET) and CSF A $\beta$. It is important to highlight that reported associations between these biomarkers (present at baseline) and FAQ scores over 36months remained significant even after adjusting for baseline RAVLT scores, suggesting that these baseline biomarkers may predict functional change independently of baseline cognitive status. No significant adjusted associations between baseline biomarkers and functional decline in the eMCI group were observed.

Baseline biomarker measures as a predictive factor of functional decline is a novel area of research, with very few studies having explored such relationships and of those comparable; our findings are congruent [14-16, 41-43]. Two separate studies found regional cortical thinning and decreased CSF A $\beta 1-42$ to predict IADL decline across the AD spectrum [15, 41]. Likewise, correlations between cerebral atrophy and IADL decline in MCI individuals have been reported [14], with rate of decline found to increase three fold in $A P O E-\varepsilon 4$ positive individuals [42]. Although not identified in our study, decreased hippocampal volume in MCI individuals too has been linked to reduced functional performance, compared to MCI individuals who do not exhibit this biomarker deficit [43]. However, once again baseline cognition was not controlled for. While not a primary aim, our results also replicate those found in relation to baseline biomarkers and cognitive decline. For example, a recent study found the combined temporal, lateral parietal, and 
posterior cingulate hypo metabolism seen on FDG-PET as well as CSF A $\beta 1-42$ and p-tau to be associated with cognitive decline in MCI and mild AD dementia patient groups [16].

Our data is an extension of these recent findings through the exploration of a more comprehensive range of biomarkers from the earliest AD stage, namely, individuals presenting with eMCI, a currently experimental subject group which in itself is a strength and novelty. Interestingly, there were no statistically significant associations observed between any of the investigated baseline biomarkers and functional decline in the eMCI cohort. A possible explanation for this finding could be the heterogeneous nature of both MCI and possibly more so the eMCI subjects in terms of AD related disease and symptom progression. eMCI is an experimental concept whereby likelihood of progression to AD dementia has not been extensively explored. A recent exploratory study utilising the ADNI cohort sought to define latent classes based on similar growth patterns between cognitive and functional decline using Growth Mixture Modelling (GMM) or person-centred modelling approach to identify baseline risk characteristics associated with the specified trajectories [44]. In doing so, they were able to create a decision tree using clinical predictors to ascertain GMM determined trajectories. Three trajectory classes (C) were identified; C1 (steepest decline), C2 (intermediate) and C3 (shallow decline). Of notable differences between $\mathrm{C} 1$ and $\mathrm{C} 3$ were the mean age $(\mathrm{C} 1=74.7, \mathrm{C} 3=72.9), A P O E \varepsilon 4$ carriage $(\mathrm{C} 1$ $=69.1 \%, \mathrm{C} 3=38.3 \%)$ and amyloid status $(\mathrm{C} 1=92 \%, \mathrm{C} 3=48.2 \%)$. Differences in these same risk variables between the eMCI and MCI cohorts explored in this study are congruent to $\mathrm{C} 3$ and $\mathrm{C} 1$ group characteristics respectively, potentially explaining the reduced likelihood of functional decline in the eMCI cohort. Indeed both cognitive and functional decline was steeper in the $\mathrm{C} 1$ group versus the $\mathrm{C} 3$ group. A longer follow-up period may bring about observed functional decline based on baseline AD-biomarker positivity in the eMCI cohort, if this data were available.

To our knowledge this is the first study to explore the association between baseline biomarker measures and functional decline independently of baseline cognitive performance and 
$A P O E \varepsilon 4$ status. Our study suggests a significant predictive value for baseline biomarkers on functional decline in MCI individuals, particularly with measures such as glucose metabolism (FDG-PET) and CSF A $\beta$ (see Supplementary Figure 1). According to the current hypothetical model of AD related biomarker trajectories [45], reduced cerebral glucose metabolism as shown in FDG PET and lower CSF A $\beta$ concentrations are all biomarker changes known to manifest during MCI disease stage. Such predictors are associated with conversion and likely reflect disease severity, i.e., how close an individual is to a significant clinical transition [45]. Hence, it may be expected that the associations are stronger for baseline FDG-PET and CSF A $\beta$ measures with functional decline due to these biomarkers being a manifestation of a disease stage involving conversion from MCI to $\mathrm{AD}$, and hence a natural follow-on of functional decline would be expected within this 36-month follow-up period.

Study limitations must be acknowledged; it is important to mention that the standardisation of biomarker cut-offs is currently limited and results often vary among laboratories. Ultimately, it will be necessary to interpret biomarker data in the context of well-established normative values. Positive or abnormal values should fall within reliable and valid pathological ranges. However, current cut-offs have been used in numerous ADNI studies, and appear to show reasonable validity for the purposes of this paper [24-26, 32, 33, 46]. Additionally, the ADNI cohort is namely white, middle class, educated and without any major comorbidity, thus, it would be important to repeat such a study with a larger and more widely represented demographic. In addition, our analyses do not take into account biomarker changes over time as AD pathophysiology progresses, which impacts on the association of biomarkers and cognitive as well as functional abilities at any given point in time during the course of $\mathrm{AD}$. Furthermore, we did not explore the effects of comorbidities and non-AD (e.g. cerebrovascular) brain changes on the studied clinical parameters. As a result, the lack of histopathological verification of the clinical diagnoses is another limitation, but the ADNI cohort is on purpose enriched with probable pre-dementia AD cases, evidenced by 
the first autopsy studies [47]. In regards to the exploratory nature of this study, multiplicity was not taken into consideration, hence it is prudent to recognise that caution must be taken when reviewing the significant outcomes of this analysis, noting the observed exploratory correlations may indicate true correlations, whereby confidence in such inferences are strengthened by congruent findings elsewhere as discussed in the manuscript [37]. Certainly there is value in running a confirmatory study, where outcomes would be sufficiently informative whether or not significance is reached. Finally, there is some missing data, especially in later visit time-points as study participants drop off. This is a classic pattern observed in prospective clinical research, and more studies surrounding participant retention across the AD spectrum is needed.

In light of next steps, exploring IADL sub-category scores by dichotomizing into complex vs simple functional tasks for added sensitivity may yield stronger correlations with baseline biomarker measures. This may also assist in validating complex IADL change as a further clinical characteristic observed in eMCI. Studies have indeed found increased sensitivity of IADL scoring in the MCI demographic, once scores are split into complex and simple categories [48, 49]. Furthermore, it may be more practical to utilise a combination of biomarkers, potentially with different weights, than individual markers to predict functional decline, and the validation of such a composite biomarker index is another important step. Finally, there is a growing interest in the literature relating to $\mathrm{AD}$ plasma biomarkers and functional decline whereby further exploration is warranted and may bring about production of invaluable confirmatory studies.

\section{Conclusion}

Patient behavioural changes and inability to perform daily functional tasks are directly related to caregiver burden, and as such provide a more meaningful construct to caregivers, practitioners, and payers [50]. Hence, it is critical that functional decline be more fully understood in relation to disease stage and correlations with well-known AD biomarkers. These results yield promise in 
supporting postulations that baseline biomarker measures may prove a significant indicator of projected functional decline, hence strengthening efficacy in utilising functional decline as a coprimary endpoint in AD clinical research.

\section{Acknowledgements}

Data collection and sharing for this project was funded by the Alzheimer's Disease Neuroimaging Initiative (ADNI) (National Institutes of Health Grant U01 AG024904) and DOD ADNI (Department of Defence award number W81XWH-12-2-0012). ADNI is funded by the National Institute on Aging, the National Institute of Biomedical Imaging and Bioengineering, and through generous contributions from the following: AbbVie, Alzheimer's Association; Alzheimer's Drug Discovery Foundation; Araclon Biotech; BioClinica, Inc.; Biogen; Bristol-Myers Squibb Company; CereSpir, Inc.; Eisai Inc.; Elan Pharmaceuticals, Inc.; Eli Lilly and Company; EuroImmun; F. Hoffmann-La Roche Ltd and its affiliated company Genentech, Inc.; Fujirebio; GE Healthcare; IXICO Ltd.; Janssen Alzheimer Immunotherapy Research \& Development, LLC.; Johnson \& Johnson Pharmaceutical Research \& Development LLC.; Lumosity; Lundbeck; Merck \& Co., Inc.; Meso Scale Diagnostics, LLC.; NeuroRx Research; Neurotrack Technologies; Novartis Pharmaceuticals Corporation; Pfizer Inc.; Piramal Imaging; Servier; Takeda Pharmaceutical Company; and Transition Therapeutics. The Canadian Institutes of Health Research is providing funds to support ADNI clinical sites in Canada. Private sector contributions are facilitated by the Foundation for the National Institutes of Health (www.fnih.org). The grantee organisation is the Northern California Institute for Research and Education, and the study is coordinated by the Alzheimer's Disease Cooperative Study at the University of California, San Diego. ADNI data are disseminated by the Laboratory for Neuro Imaging at the University of Southern California. 
Robb et al.: Biomarkers and ADL in AD

Conflict of interest statement: The authors have no conflict of interest to report.

Author contributions: Study concept and design: Robb, Udeh-Momoh, Alexopoulos, and Perneczky. Analysis and interpretation of data: Robb, Udeh-Momoh, Perneczky. Drafting of the manuscript: Robb, Udeh-Momoh, and Perneczky. Critical revision of the manuscript for important intellectual content: Robb, Udeh-Momoh, Wagenpfeil, Schöpe, Alexopoulos, Perneczky. Statistical analysis: Wagenpfeil, Schöppe. Administrative, technical, and material support: Alexopoulos, Perneczky. Study supervision: Perneczky. 
Robb et al.: Biomarkers and ADL in AD

\section{References}

[1] Luck T, Luppa M, Riedel-Heller MC, Luck A, Angermeyer HH, Angermeyer SG, Villringer SG, König SG (2011) Impact of impairment in instrumental activities of daily living and mild cognitive impairment on time to incident dementia: Results of the Leipzig Longitudinal Study of the Aged. Psychol Med 41, 1087-1097.

[2] Tabert MH, Borukhova-Milov SM, Camacho L, Pelton Y, Liu G, Devanand X, Tabert Y, Albert DP, Pelton DP, Stern DP, Devanand DP, Tabert DP (2002) Functional deficits in patients with mild cognitive impairment: Prediction of AD. Neurology 58, 758-764.

[3] Nygård L (2003) Instrumental activities of daily living: a stepping-stone towards Alzheimer's disease diagnosis in subjects with mild cognitive impairment? Acta Neurol Scand 107, 42-46.

[4] Amieva H, Mokri H, Le Goff M, Meillon C, Jacqmin-Gadda H, Foubert-Samier A, Orgogozo J, Stern Y, Dartigues J (2014) Compensatory mechanisms in higher- educated subjects with Alzheimer's disease: a study of 20 years of cognitive decline. Brain 137, 1167-1175.

[5] McKhann GM, Knopman DS, Chertkow H, Hyman BT, Jack CR, Jr., Kawas CH, Klunk WE, Koroshetz WJ, Manly JJ, Mayeux R, Mohs RC, Morris JC, Rossor MN, Scheltens P, Carrillo MC, Thies B, Weintraub S, Phelps CH (2011) The diagnosis of dementia due to Alzheimer's disease: recommendations from the National Institute on Aging-Alzheimer's Association workgroups on diagnostic guidelines for Alzheimer's disease. Alzheimers Dement 7, 263-269.

[6] Aisen PS, Walter S, Petersen RC, Donohue MC, Gamst A, Raman R, Thomas RG, Trojanowski JQ, Shaw LM, Beckett LA, Jack Jr CR, Jagust W, Toga AW, Saykin AJ, Morris JC, Green RC, Weiner MW (2010) Clinical core of the Alzheimer's disease neuroimaging initiative: Progress and plans. Alzheimers Dement 6, 239-246. 
Robb et al.: Biomarkers and ADL in AD

[7] Sperling RA, Aisen PS, Beckett LA, Bennett DA, Craft S, Fagan AM, Iwatsubo T, Jack CR, Jr., Kaye J, Montine TJ, Park DC, Reiman EM, Rowe CC, Siemers E, Stern Y, Yaffe K, Carrillo MC, Thies B, Morrison-Bogorad M, Wagster MV, Phelps CH (2011) Toward defining the preclinical stages of Alzheimer's disease: recommendations from the National Institute on Aging-Alzheimer's Association workgroups on diagnostic guidelines for Alzheimer's disease. Alzheimers Dement 7, 280-292.

[8] Albert MS, DeKosky ST, Dickson D, Dubois B, Feldman HH, Fox NC, Gamst A, Holtzman DM, Jagust WJ, Petersen RC, Snyder PJ, Carrillo MC, Thies B, Phelps CH (2011) The diagnosis of mild cognitive impairment due to Alzheimer's disease: recommendations from the National Institute on Aging-Alzheimer's Association workgroups on diagnostic guidelines for Alzheimer's disease. Alzheimers Dement 7, 270279.

[9] McConathy J, Sheline YI (2014) Imaging Biomarkers Associated With Cognitive Decline: A Review. Biol Psychiatry, 77, 685-689.

[10] Lo RY, Hubbard AE, Shaw LM, Trojanowski JQ, Petersen RC, Aisen PS, Weiner MW, Jagust WJ, Alzheimer's Disease Neuroimaging I (2011) Longitudinal change of biomarkers in cognitive decline. Arch Neurol 68, 1257-1266.

[11] Gomar J, Conejero-Goldberg C, Davies P, Goldberg T (2014) Extension and refinement of the predictive value of different classes of markers in ADNI: Four- year follow- up data. Alzheimers. Dement. 10, 704-712.

[12] Kozauer N, Katz R (2013) Regulation of drugs for early Alzheimer's disease. N Engl J Med 369, 288.

[13] Marshall G, Olson L, Frey M, Maye J, Becker J, Rentz DM, Sperling R, Johnson K (2011) Instrumental Activities of Daily Living Impairment Is Associated with Increased Amyloid Burden. Dement Geriatr Cogn Disord 31, 443-450. 
[14] Okonkwo OC, Alosco ML, Jerskey BA, Sweet LH, Ott BR, Tremont G (2010) Cerebral atrophy, apolipoprotein E varepsilon4, and rate of decline in everyday function among patients with amnestic mild cognitive impairment. Alzheimers Dement, 6, 404.

[15] Okonkwo OC, Mielke MM, Alosco ML, Tremont G, Griffith HR, Shaw LM, Trojanowski JQ (2010) Cerebrospinal fluid abnormalities and rate of decline in everyday function across the dementia spectrum: Normal aging, mild cognitive impairment, and Alzheimer disease. Arch Neurol 67, 688-696.

[16] Landau SM, Harvey D, Madison CM, Koeppe RA, Reiman EM, Foster NL, Weiner MW, Jagust WJ (2011) Associations between cognitive, functional, and FDG- PET measures of decline in AD and MCI. Neurobiol Aging 32, 1207-1218.

[17] A. R (1964) PUF, Paris, France.

[18] Pfeffer RI, Kurosaki TT, Harrah CH, Jr., Chance JM, Filos S (1982) Measurement of functional activities in older adults in the community. J Gerontol 37, 323-329.

[19] Blacker D, Lee H, Muzikansky A, Martin EC, Tanzi R, McArdle JJ, Moss M, Albert M (2007) Neuropsychological measures in normal individuals that predict subsequent cognitive decline. Arch Neurol 64, 862.

[20] Devanand DP, Liu X, Tabert MH, Pradhaban G, Cuasay K, Bell K, de Leon MJ, Doty RL, Stern Y, Pelton GH (2008) Combining Early Markers Strongly Predicts Conversion from Mild Cognitive Impairment to Alzheimer's Disease. Biol Psychiatry 64, 871-879.

[21] Dickerson BC, Sperling RA, Hyman BT, Albert MS, Blacker D (2007) Clinical prediction of Alzheimer disease dementia across the spectrum of mild cognitive impairment. Arch Gen Psychiat 64, 1443.

[22] Gomar JJ, Bobes-Bascaran MT, Conejero-Goldberg C, Davies P, Goldberg TE (2011) Utility of combinations of biomarkers, cognitive markers, and risk factors to predict 
conversion from mild cognitive impairment to Alzheimer disease in patients in the Alzheimer's disease neuroimaging initiative. Arch Gen Psychiat 68, 961.

[23] Teng E, Becker BW, Woo E, Knopman DS, Cummings JL, Lu PH (2010) Utility of the functional activities questionnaire for distinguishing mild cognitive impairment from very mild Alzheimer disease. Alzheimer Dis Assoc Disord 24, 348-353.

[24] Landau SM, Harvey D, Madison CM, Reiman E, Foster N, Aisen PS, Petersen RC, Shaw L, Trojanowski J, Jack CR, Weiner M, Jagust W (2010) Comparing predictors of conversion and decline in mild cognitive impairment. Neurology 75, 230-238.

[25] Landau SM, Jagust WJ, Mintun MA, Joshi AD, Koeppe RA, Petersen RC, Aisen PS, Weiner MW (2012) Amyloid deposition, hypometabolism, and longitudinal cognitive decline. Ann Neurol 72, 578-586.

[26] Jagust W, Landau SM, Shaw L, Trojanowski J, Koeppe R, Reiman E, Foster N, Petersen RC, Weiner M, Price J, Mathis C (2009) Relationships between biomarkers in aging and dementia. Neurology 73, 1193-1199.

[27] Jack CR, Jr., Bernstein MA, Fox NC, Thompson P, Alexander G, Harvey D, Borowski B, Britson PJ, J LW, Ward C, Dale AM, Felmlee JP, Gunter JL, Hill DL, Killiany R, Schuff N, Fox-Bosetti S, Lin C, Studholme C, DeCarli CS, Krueger G, Ward HA, Metzger GJ, Scott KT, Mallozzi R, Blezek D, Levy J, Debbins JP, Fleisher AS, Albert M, Green R, Bartzokis G, Glover G, Mugler J, Weiner MW (2008) The Alzheimer's Disease Neuroimaging Initiative (ADNI): MRI methods. J Magn Reson Imaging 27, 685-691.

[28] Bonner-Jackson A, Okonkwo O, Tremont G, Alzheimer's Disease Neuroimaging I (2012) Apolipoprotein E epsilon2 and functional decline in amnestic mild cognitive impairment and Alzheimer disease. Am J Geriatr Psychiatry 20, 584-593.

[29] Voevodskaya O, Simmons A, Nordenskjold R, Kullberg J, Ahlstrom H, Lind L, Wahlund LO, Larsson EM, Westman E, Alzheimer's Disease Neuroimaging I (2014) The effects of 
intracranial volume adjustment approaches on multiple regional MRI volumes in healthy aging and Alzheimer's disease. Front Aging Neurosci 6, 264.

[30] Kim S, Swaminathan S, Shen L, Risacher SL, Nho K, Foroud T, Shaw LM, Trojanowski JQ, Potkin SG, Huentelman MJ, Craig DW, DeChairo BM, Aisen PS, Petersen RC, Weiner MW, Saykin AJ, Alzheimer's Disease Neuroimaging I (2011) Genome-wide association study of CSF biomarkers Abeta1-42, t-tau, and p-tau181p in the ADNI cohort. Neurology 76, 69-79.

[31] Steenland K, Zhao L, Goldstein F, Cellar J, Lah J (2014) Biomarkers for predicting cognitive decline in those with normal cognition. J Alzheimers Dis 40, 587-594.

[32] Shaw L, Vanderstichele H, Knapik-Czajka M, Clark CM, Aisen PS, Petersen RC, Blennow K, Soares H, Simon A, Lewczuk P, Dean R, Siemers E, Potter W, Lee V, Trojanowski J (2009) Cerebrospinal Fluid Biomarker Signature in Alzheimer's Disease Neuroimaging Initiative Subjects. Ann Neurol 65, 403-413.

[33] Landau SM, Lu M, Joshi AD, Pontecorvo M, Mintun MA, Trojanowski JQ, Shaw LM, Jagust WJ (2013) Comparing positron emission tomography imaging and cerebrospinal fluid measurements of beta-amyloid. Ann Neurol 74, 826-836.

[34] Zeger SL, Liang KY (1986) Longitudinal data analysis for discrete and continuous outcomes. Biometrics 42, 121.

[35] Hubbard AE, Ahern J, Fleischer NL, Van Der Laan M, Lippman SA, Jewell N, Bruckner T, Satariano WA (2010) To GEE or not to GEE: comparing population average and mixed models for estimating the associations between neighborhood risk factors and health. Epidemiology (Cambridge, Mass.) 21, 467.

[36] Perneczky R, Pohl C, Sorg C, Hartmann J, Komossa K, Alexopoulos P, Wagenpfeil S, Kurz A (2006) Complex activities of daily living in mild cognitive impairment: conceptual and diagnostic issues. Age Ageing 35, 240-245. 
Robb et al.: Biomarkers and ADL in AD

[37] Bender R, Lange S (2001) Adjusting for multiple testing — when and how? J Clin Epidemiol 54, 343-349.

[38] Wickham H, ggplot2: Elegant graphics for data analysis., http://cran.rproject.org/package=ggplot 2.

[39] Team RC, R: A language and environment for statistical computing, http://www.rproject.org/.

[40] Carey V, gee: Generalised estimation equation solver (ported to R by Lunley, T. \& Ripley, B.), http://cran.r-project.org/package=gee.

[41] Marshall GA, Lorius N, Locascio JJ, Hyman BT, Rentz DM, Johnson KA, Sperling RA, Initi AsDN (2014) Regional Cortical Thinning and Cerebrospinal Biomarkers Predict Worsening Daily Functioning Across the Alzheimer's Disease Spectrum. J Alzheimers Dis 41, 719-728.

[42] Albert SM, Gurland B, Maestre G, Jacobs DM, Stern Y, Mayeux R (1995) APOE genotype influences functional status among elderly without dementia. Am J Med Genet 60, 583587.

[43] Brown P, Devanand D, Liu X, Caccappolo E (2011) Functional Impairment in Elderly Patients With Mild Cognitive Impairment and Mild Alzheimer Disease. Arch Gen Psychiat 68, 617-626.

[44] Hochstetler H, Trzepacz PT, Wang S, Yu P, Case M, Henley DB, Degenhardt E, Leoutsakos JM, Lyketsos CG (2015) Empirically Defining Trajectories of Late-Life Cognitive and Functional Decline. J Alzheimers Dis 50, 271-282.

[45] Jack Jr CR, Knopman DS, Petersen RC, Jagust WJ, Shaw LM, Trojanowski JQ, Aisen PS, Weiner MW (2010) Hypothetical model of dynamic biomarkers of the Alzheimer's pathological cascade. Lancet Neurol 9, 119-128. 
Robb et al.: Biomarkers and ADL in AD

[46] Alexopoulos P, Grimmer T, Perneczky R, Kurz A, Kriett L, Klupp E, Förster S, Yakushev I, Haller B, Gray K, Laskaris N, Drzezga A, Fellgiebel A (2014) Limited agreement between biomarkers of neuronal injury at different stages of Alzheimer's disease. Alzheimers Dement, 10, 684-689.

[47] Toledo JB, Cairns NJ, Da X, Chen K, Carter D, Fleisher A, Householder E, Ayutyanont N, Roontiva A, Bauer RJ, Eisen P, Shaw LM, Davatzikos C, Weiner MW, Reiman EM, Morris JC, Trojanowski JQ, Alzheimer's Disease Neuroimaging I (2013) Clinical and multimodal biomarker correlates of ADNI neuropathological findings. Acta Neuropathol Commun 1, 65.

[48] Perneczky R, Pohl C, Sorg C, Hartmann J, Tosic N, Grimmer T, Heitele S, Kurz A (2006) Impairment of activities of daily living requiring memory or complex reasoning as part of the MCI syndrome. Int J Geriatr Psychiatry 21, 158-162.

[49] Hesseberg K, Bentzen H, Ranhoff AH, Engedal K, Bergland A (2013) Disability in instrumental activities of daily living in elderly patients with mild cognitive impairment and Alzheimer's disease. Dement Geriatr Cogn Disord 36, 146-153.

[50] Bergvall N, Brinck P, Eek D, Gustavsson A, Wimo A, Winblad B, Jonsson L (2011) Relative importance of patient disease indicators on informal care and caregiver burden in Alzheimer's disease. Int Psychogeriatr 23, 73-85. 
Robb et al.: Biomarkers and ADL in AD

Table 1. Description of the study sample

\begin{tabular}{|c|c|c|c|c|}
\hline & \multicolumn{4}{|c|}{ Diagnostic group } \\
\hline & $\begin{array}{l}\text { Overall } \\
(\mathrm{N}=509)\end{array}$ & $\begin{array}{l}\text { Normal } \\
(n=156)\end{array}$ & $\begin{array}{c}\text { eMCl } \\
(n=189)\end{array}$ & $\begin{array}{c}\mathrm{MCl} \\
(\mathrm{n}=164)\end{array}$ \\
\hline Age, mean $\pm S D$ & $73.2 \pm 7.1$ & $74.8 \pm 5.5$ & $71.2 \pm 7.5$ & $74.0 \pm 7.4$ \\
\hline Gender, male (\%) & 58.0 & 57.7 & 55.0 & 61.6 \\
\hline \multicolumn{5}{|l|}{$\overline{F A Q}$, Median (IQR) } \\
\hline Baseline & $0.0(3.0)^{*}$ & $0.0(0.0)$ & $1.0(3.0)$ & $2.0(6.0)^{*}$ \\
\hline Month 12 & $0.0(4.0)^{\star}$ & $0.0(0.0)^{*}$ & $1.0(4.0)^{*}$ & $4.0(9.0)^{*}$ \\
\hline Month 24 & $1.0(4.0)^{*}$ & $0.0(0.0)^{*}$ & $1.0(3.0)^{*}$ & $6.0(12.0)^{*}$ \\
\hline Month 36 & $2.0(8.0)^{*}$ & $0.0(0.75)^{*}$ & $1.0(4.0)^{*}$ & $8.5(16.0)^{*}$ \\
\hline \multicolumn{5}{|l|}{ RAVLT, Median (IQR) } \\
\hline Baseline & $37.0(16.0)$ & $44.0(15.0)$ & $37.0(15.0)$ & $30.0(12.0)$ \\
\hline 12 & $37.0(16.25)^{\star}$ & $43.0(14.0)^{*}$ & $38.0(14.0)^{*}$ & $29.0(11.0)^{*}$ \\
\hline 24 & $37.0(18.0)^{\star}$ & $44.5(14.25)^{*}$ & $37.0(16.5)^{*}$ & $28.0(13.0)^{*}$ \\
\hline 36 & $34.0(16.0)^{\star}$ & $39.0(10.5)^{\star}$ & $37.0(13.75)^{\star}$ & $24.0(12.0)^{*}$ \\
\hline \multicolumn{5}{|l|}{$\begin{array}{l}\text { Biomarkers, AD-positive } \\
\text { (abnormal) (\%) }\end{array}$} \\
\hline$A P O E \varepsilon 4$ & 39.1 & 23.1 & 38.6 & 54.9 \\
\hline FDG-PET (count value & 32.4 & 19.9 & 24.3 & 53.7 \\
\hline$\leq 1.21)$ & & & & \\
\hline $\begin{array}{l}\text { MRI hippocampal } \\
\text { volume ( } \leq 3260 \mathrm{~mm} 3)\end{array}$ & 30.5 & 16.0 & 23.3 & 52.4 \\
\hline CSF Aß1-42 (s192 pg/ml) & 47.0 & 32.7 & 36.0 & 73.2 \\
\hline CSF p-tau181 (>23 pg/ml) & 46.6 & 33.3 & 38.1 & 68.9 \\
\hline CSF ttau ( $\geq 66 \mathrm{pg} / \mathrm{ml})$ & 30.4 & 21.6 & 24.6 & 45.4 \\
\hline
\end{tabular}

*: based on available cases specified (FAQ and/or AVLT scores were not ascertained at missed visit time-points); IQR: Interquartile Range; eMCI: early Mild Cognitive Impairment; MCI: Mild Cognitive Impairment; FAQ: Functional activity questionnaire (higher scores indicate greater functional impairment); RAVLT: Rey auditory verbal learning test (lower scores indicate greater memory impairment); Positive (abnormal): baseline biomarkers which measured within the pathological range; Negative (normal): baseline biomarkers which measured within the normal range; APOE: Apolipoprotien E; CSF: cerebrospinal fluid; FDG PET: [18F] fluorodeoxyglucose positron emission tomography; p-tau181: tau phosphorylated at threonine 181; $\mathrm{A} \beta(+)$ : participants with $\beta$ - amyloid 1-42 levels in cerebrospinal fluid (CSF) lower $\leq 192 \mathrm{pg} / \mathrm{ml}$. 
Robb et al.: Biomarkers and ADL in AD

Table 2. Associations between FAQ and RAVLT progression with baseline AD-risk biomarkers in normal, eMCI and MCI participants

\begin{tabular}{|c|c|c|c|c|}
\hline & RAVLT & & FAQ & \\
\hline AD-Positive Biomarkers & $\begin{array}{r}\text { Regression } \\
\text { coefficient }\end{array}$ & $\begin{array}{c}p \text {-value } \\
(\text { sig.<0.05) }\end{array}$ & $\begin{array}{l}\text { Regression } \\
\text { coefficient }\end{array}$ & $\begin{array}{c}p \text {-value } \\
\text { (sig. }<0.05)\end{array}$ \\
\hline \multicolumn{5}{|l|}{$\begin{array}{l}\text { FDG-PET (count value } \\
\leq 1.21 \text { ) }\end{array}$} \\
\hline Normal & -3.680 & 0.620 & 0.242 & 1.000 \\
\hline eMCl & -0.027 & 1.000 & 1.382 & 0.426 \\
\hline $\mathrm{MCl}$ & -4.605 & $0.009^{*}$ & 3.592 & $<0.001^{*}$ \\
\hline \multicolumn{5}{|l|}{$\begin{array}{l}\text { MRI hippocampal volume } \\
(\leq 3260 \mathrm{~mm} 3)\end{array}$} \\
\hline Normal & 1.227 & 1.000 & 0.769 & 1.000 \\
\hline $\mathrm{eMCl}$ & -0.623 & 1.000 & 1.578 & 0.222 \\
\hline $\mathrm{MCl}$ & -1.584 & 1.000 & 2.221 & 0.161 \\
\hline \multicolumn{5}{|l|}{ CSF Aß1-42 (s192 pg/ml) } \\
\hline Normal & 0.200 & 1.000 & 0.117 & 1.000 \\
\hline eMCl & -3.777 & 0.063 & 1.056 & 0.636 \\
\hline $\mathrm{MCl}$ & -6.594 & $0.005^{\star}$ & 2.972 & $0.028^{*}$ \\
\hline \multicolumn{5}{|l|}{ CSF p-tau181 (>23 pg/ml) } \\
\hline Normal & 0.343 & 1.000 & 0.333 & 1.000 \\
\hline $\mathrm{eMCl}$ & -0.835 & 1.000 & 0.419 & 1.000 \\
\hline $\mathrm{MCl}$ & -6.850 & $<0.001^{*}$ & 0.421 & 1.000 \\
\hline \multicolumn{5}{|l|}{ CSF ttau ( $\geq 66 \mathrm{pg} / \mathrm{ml})$} \\
\hline Normal & -1.741 & 1.000 & 0.804 & 1.000 \\
\hline $\mathrm{eMCl}$ & -0.666 & 1.000 & 1.083 & 1.000 \\
\hline $\mathrm{MCl}$ & -3.279 & 0.236 & -0.339 & 1.000 \\
\hline
\end{tabular}

*: $\mathrm{p} \leq 0.05$ (GEE models were adjusted for age, $A P O E \varepsilon 4$, education, and respectively FAQ (GEE models for RAVLT) or RAVLT (GEE models for FAQ) at baseline)

eMCI: early Mild Cognitive Impairment; MCI: Mild Cognitive Impairment; FAQ: Functional activity questionnaire; RAVLT: Rey auditory verbal learning test; Positive (abnormal): baseline biomarkers which measured within the pathological range; Negative (normal): baseline biomarkers 
Robb et al.: Biomarkers and ADL in AD

which measured within the normal range; APOE: Apolipoprotien E; CSF: cerebrospinal fluid; FDG PET: [18F] fluorodeoxyglucose positron emission tomography; $\mathrm{p}$-tau181: tau phosphorylated at threonine 181; t-tau: total tau measurement; $\operatorname{CSF} \mathrm{A} \beta(+)$ : participants with $\beta$ - amyloid 1-42 levels in cerebrospinal fluid (CSF) lower $\leq 192 \mathrm{pg} / \mathrm{ml}$. 
Robb et al.: Biomarkers and ADL in AD

Supplementary Figure 2. Temporal representation of RAVLT composite measurements in normal, eMCI and MCI subjects according to baseline biomarker profiles (raw and unadjusted values).

eMCI: early Mild Cognitive Impairment; MCI: Mild Cognitive Impairment; FAQ: Functional activity questionnaire; abnormal: baseline biomarkers which measured within the pathological range; normal: baseline biomarkers which measured within the normal range; CSF: cerebrospinal fluid; FDG PET: [18F] fluorodeoxyglucose positron emission tomography; p-tau181: tau phosphorylated at threonine 181; t-tau: total tau measurement; CSF A $\beta$ positive: participants with $\beta$-amyloid 1-42 levels in cerebrospinal fluid (CSF) lower $\leq 192 \mathrm{pg} / \mathrm{ml}$. 